\title{
Nano iron (Fe) complex is an effective source of Fe for tobacco plants grown under low Fe supply
}

\author{
Soodabeh Bastani ${ }^{2}$, Roghieh Hajiboland ${ }^{1,2^{*}}$, Masoumeh Khatamian ${ }^{3}$, Maryam Saket- \\ Oskoui $^{3}$
}

${ }^{1}$ Center of Excellence for Biodiversity, University of Tabriz, 51666-14779 Iran. ${ }^{2}$ Department of Plant Science, University of Tabriz, 51666-14779, Iran. ${ }^{3}$ Department of Inorganic Chemistry, University of Tabriz, 5166614779, Iran. *Corresponding author: ehsan@tabrizu.ac.ir

\begin{abstract}
In order to compare the uptake, utilization and distribution of iron (Fe) as nano and bulk Fe complex (Fe(III)EDTA), an experiment was conducted using hydroponically-grown Fe-deficient tobacco (Nicotiana rustica L.) plants. Plants were treated with Fe either through roots (root application, RA) or leaves (foliar application, FA). Leaf chlorophyll concentration and plants biomass responded to the Fe re-supply; this response was quicker for the nano Fe complex than the bulk Fe complex, in particular, in the RA plants. Plants re-supplied with the nano Fe complex had lower Fe content but higher Fe use efficiency than plants re-supplied with the bulk Fe complex. Analysis of different plant fractions at two subsequent weeks revealed that the nano Fe complex had higher mobility than the bulk Fe complex, both in the xylem and the phloem. Comparison of RA and FA revealed that the re-supply of Fe through roots was more efficient in retrieving the whole plant growth than the foliar Fe spray. RA, in contrast to FA plants, profited from an enhanced Fe uptake capacity induced under Fe starvation in the roots. Alternatively, FA was effective in extending the green leaf area duration. Our data suggested that nano Fe complex is advantageous as both leaf spray and a long-term feeding of plant through roots.
\end{abstract}

Keywords: Utilization, efficiency, partitioning, retranslocation, leaf age 


\section{Introduction}

Iron $(\mathrm{Fe})$ is one of the essential micronutrients for plant growth and development and is required for a wide range of biochemical processes from photosynthesis to respiration. Fe is essential for maintaining the chloroplast structure and function, for biosynthesis of Fe-S clusters and chlorophyll (Chl), and is involved in the electron transport systems (Broadley et al., 2012; Briat et al., 2015; Mai et al., 2016).

$\mathrm{Fe}$ is the third most limiting nutrient for plant growth. Chlorosis of young leaves is often the first visual sign of Fe deficiency and is not only associated with the loss of Chl but also with changes in the expression and assembly of other components of the photosynthetic apparatus (Broadley et al., 2012; Mai et al., 2016). The application of Fe fertilizers to the soil is widely practised in cropping systems throughout the world in order to reduce the deficiency symptoms and for biofortification purposes (Márquez-Quiroz et al., 2015); however, Fe fertilizers are only scantily available to the plant roots (Zuo and Zhang, 2011; Rengel, 2015).

In aerobic soil, the majority of $\mathrm{Fe}$ is converted to the oxidised ferric form, which is extremely immobile in soil, leading to Fe deficiency in plants. In addition, soil $\mathrm{pH}$ in the range of 7.4-8.5 causes low solubility and slow dissolution kinetics of Fe-bearing minerals (Granja and Covarrubias, 2018). Also elevated bicarbonate concentrations, typical of calcareous soils, reduce the $\mathrm{Fe}$ acquisition by plants. Under these conditions, lime-induced chlorosis occurs in sensitive crops as a consequence of limited bioavailability of $\mathrm{Fe}$ in the soil and the impaired acquisition and translocation of Fe by the plants (Lucena and HernandezApaolaza, 2017). To resolve this issue, supply of Fe using foliar sprays has been practised in many agricultural systems (Kannan, 2010; Yadegari, 2015).
Foliar fertilization facilitates the rapid absorption of mineral elements, and can be carried out throughout the growing season, in particular, during peak periods of nutritional demand without interaction with the soil components, precipitation, adsorption onto soil surfaces or risk of loss by erosion (Fernández and Brown, 2013). Size, hydrophilicity, lipophilicity, and net charge are features of the applied materials that considerably affect the penetration of foliar fertilizers (Eichert and Fernández, 2012). Nutrient uptake may be enhanced by reducing the particle size that increases the specific surface area of a fertilizer, thus increasing its contact with plant surfaces.

Foliar Fe fertilization is an economic and target-oriented approach to cure $\mathrm{Fe}$ chlorosis in crop plants. Foliar uptake is a pre-requisite for the efficient metabolic use of the Fe applied to the leaves. A re-greening effect due to enhanced $\mathrm{Chl}$ concentration after $\mathrm{Fe}$ supply to the leaves is a main indicator of efficient foliar uptake of the applied Fe-fertilizer. Variable Fe and surfactant concentrations have been supplied to plants. The use of highly concentrated solutions may cause leaf burn and defoliation (Fernandez and Ebert, 2005). Previous studies stated that the penetration of a leaf-applied Fe-containing solution is affected by several plant-related, environmental and physicochemical factors. In consequence, the response to $\mathrm{Fe}$ sprays is immensely variable depending on the plant species and experimental conditions (Fernandez and Ebert, 2005). Foliar Fe fertilization studies are currently focused on product formulation-dependent $\mathrm{Fe}$ uptake and use efficiencies. Major targets are, among others, Fe oxidation states, ions vs. chelates, $\mathrm{pH}$, surfactants and adjuvants, as well as mechanisms and pathways of Fe uptake into the leaf (Rios et al., 2016). Considering the complexity of penetration, translocation and bioavailability of leaf-applied Fe, Fe foliar 
sprays are yet to be successfully formulated (Fernandez et al., 2008). Collecting relevant information on Fe transport and partitioning after foliar application, will facilitate the selection of suitable Fe sprays.

Nanoparticles (NPs) are atomic or molecular aggregates with at least one dimension between 1 and 100 $\mathrm{nm}$. These particles exhibit novel properties based on specific characteristics such as size, distribution and morphology (Monreal et al., 2015; Tripathi et al., 2017). A literature review on studies concerning the application of Fe NPs to plants reveals that most experiments focus on the feasibility of Fe absorption by roots or leaves, the accumulation of Fe in the vegetative parts and seeds, and the probable growth effects. The efficiency of Fe oxide $\left(\mathrm{Fe}_{3} \mathrm{O}_{4}\right)$ NPs as Fe fertilizer depends on both the plant species and the growth substrate used in the experiment (Zhu et al., 2008). In maize, addition of $\mathrm{Fe}$ oxide $\left(\mathrm{Fe}_{3} \mathrm{O}_{4}\right)$ NPs through leaves stimulated growth, $\mathrm{Chl}$ and $\mathrm{Fe}$ and ferritin concentrations (Jalali et al., 2016). In spinach, application of $\mathrm{Fe}_{3} \mathrm{O}_{4}$ NPs via hydroponics increased growth rate and productivity of plants due to the uptake of $\mathrm{Fe}_{3} \mathrm{O}_{4}$ (Jeyasubramanian et al., 2016). Application of different sources of Fe NPs including $\mathrm{Fe}_{3} \mathrm{O}_{4}$ NPs, monodisperse $\mathrm{Fe}_{3} \mathrm{O}_{4}$ NPs, polymeric $\mathrm{Fe}_{3} \mathrm{O}_{4}$ NPs and polymeric monodisperse $\mathrm{Fe}_{3} \mathrm{O}_{4} \mathrm{NPs}$ in sorghum showed that the polymeric monodisperse $\mathrm{Fe}_{3} \mathrm{O}_{4}$ NPs was the most effective form of Fe NPs leading to a significant increase in the leaf $\mathrm{Chl}$ and $\mathrm{Fe}$ contents of shoot and roots (Golshahi et al., 2018).

Application of relatively high concentrations of $\mathrm{Fe}$ (100 ppm) as Fe-chelate and $\mathrm{Fe}_{3} \mathrm{O}_{4}$ NPs to the leaves of maize plants during two subsequent generations resulted in reduction of biomass and $\mathrm{Chl}$ for $\mathrm{Fe}_{3} \mathrm{O}_{4}$ NPs-treated plants in the second progeny (Jalali et al., 2017). This was consequence of the accumulation of toxic Fe levels in plants treated with $\mathrm{Fe}_{3} \mathrm{O}_{4}$ NPs. Surprisingly, none of these studies used Fe-deficient plants for the treatments, and they often applied high- er Fe concentrations than those recommended to meet the plants nutritional requirement. To the best of our knowledge, no Fe complex synthesised at nanoscale has been experimented in plants to date. Exploring new $\mathrm{Fe}$ fertilizers through nanotechnology provides alternative methods for overcoming the Fe deficiency in crop production.

Tobacco (Nicotiana spp.) is the most essential nonfood crop species worldwide and is produced in more than 100 countries under various climatic conditions with different soil types. This experiment was conducted using hydroponically-grown Fe-deficient tobacco plants in order to compare the nano and bulk Fe application effect via roots or leaves. In addition to growth, Fe utilisation efficiency and its distribution into different shoot fractions were investigated and compared between the two different Fe complexes and their application modes.

\section{Materials and Methods}

\subsection{Plants pre-culture}

Seeds of tobacco (Nicotiana rustica L.) plants were surface-sterilised using $1 \%$ active hypochlorite and germinated on perlite in dark and were moistened by distilled water. After emergence of primary leaves, the seedlings were transferred to light and were irrigated with $50 \%$ modified Hoagland nutrient solution $(\mathrm{pH}$ 5.8) consisting of macronutrients $(\mathrm{mM}): \mathrm{KNO}_{3} 6.0$, $\mathrm{Ca}\left(\mathrm{NO}_{3}\right) 2.4 \mathrm{H}_{2} \mathrm{O} 4.0, \mathrm{NH}_{4} \mathrm{H}_{2} \mathrm{PO}_{4} 2.0, \mathrm{MgSO}_{4} 1.0$ and micronutrients $(\mu \mathrm{M}): \mathrm{KCl} 50, \mathrm{H}_{3} \mathrm{BO}_{3} 25, \mathrm{MnSO}_{4} \cdot \mathrm{H}_{2} \mathrm{O}$ 2.0, $\mathrm{ZnSO}_{4} .7 \mathrm{H}_{2} \mathrm{O} 2.0, \mathrm{CuSO}_{4} .5 \mathrm{H}_{2} \mathrm{O} 0.5, \mathrm{H}_{2} \mathrm{MoO}_{4} 0.5$ and Fe(II)-EDTA 20 (Johnson et al., 1957). Thirtyday-old young seedlings were transferred to the hydroponic medium in $2 \mathrm{~L}$ plastic pots (two plants per pot) and were pre-cultured for 30 days. The nutrient solution was replaced every 6 days throughout the experiment, unless otherwise stated. 


\subsection{Synthesis of nano Fe complex}

The solution of $10 \mathrm{mM} \mathrm{Na}{ }_{2} \mathrm{H}_{2}$ EDTA. $2 \mathrm{H}_{2} \mathrm{O}$ was mixed with $1 \mathrm{M} \mathrm{NaOH}$ and heated gently until dissolved. Furthermore, $9 \mathrm{mM}$ of $\mathrm{FeCl}_{3} \cdot 6 \mathrm{H}_{2} \mathrm{O}$ was dissolved in water by simultaneous heating and stirring. Thereafter, the dissolved $\mathrm{FeCl}_{3}$-solution was slowly poured into the EDTA-solution and stirred. The final solution was gently warmed and after partial evaporation, a yellow-coloured precipitation $(\mathrm{Na}[\mathrm{Fe}(\mathrm{EDTA})])$ appeared. After cooling, the obtained product was collected by suction filtration, washed twice with ethanol and dried on filter paper at room temperature (Figure 1A). For preparation of nano $\mathrm{Fe}(\mathrm{III})$-EDTA stock solution $(5.5 \mathrm{mM}), 0.1 \mathrm{~g}$ of synthesised $\mathrm{Na}[\mathrm{Fe}$ (EDTA)] was poured in $50 \mathrm{ml}$ distilled water, stirred and heated until dissolved.

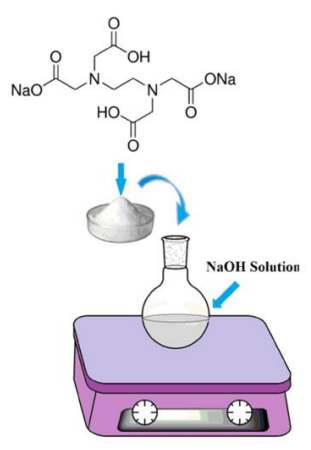

Step 1: Solving of

$\mathrm{Na}_{2} \mathrm{H}_{2}$ EDTA. $2 \mathrm{H}_{2} \mathrm{O}$

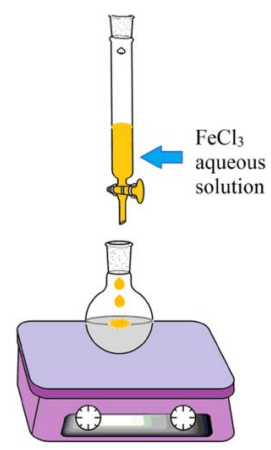

Step 2: Addition of

$\mathrm{FeCl}_{3}$ solution
Step 3: Cooling in ice bath

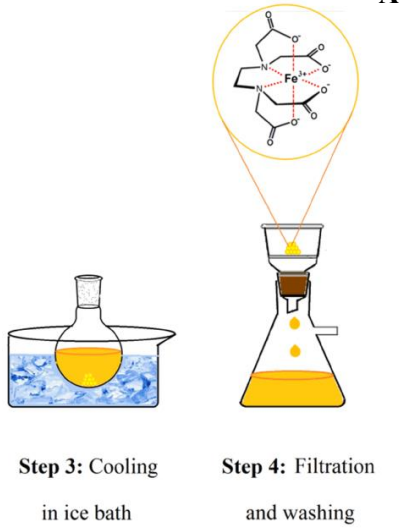

Step 4: Filtration and washing

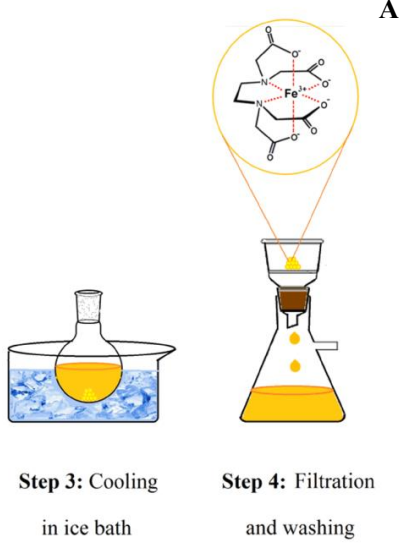

A

C
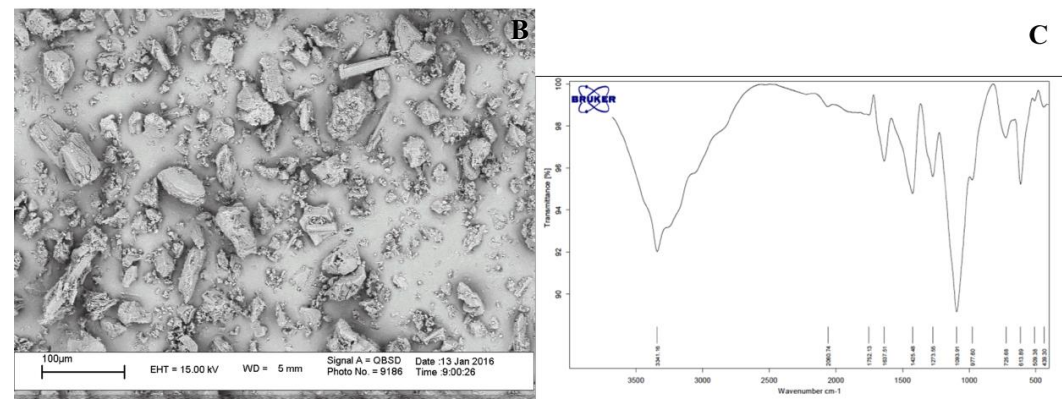

Figure 1. Schematic illustration of the synthesis of the Fe nano-complex (Na[Fe(EDTA)]) (A), scanning electron microscopy (SEM) image (B) and Fourier-transform infra-red (FT-IR) spectrophotometry (C) of the synthesized compound. 


\subsection{Structural characterization of nano Fe complex}

Structural characteristics of nanoparticles were evaluated using scanning electron microscopy (LEO, 1430VP, Carl Zeiss, Oberkochen, Germany) and Fouriertransform infra-red spectroscopy (FT-IR, Bruker IR-spectrometer, Tensor 27, Bruker, Germany). According to the SEM image, nano-Na[Fe(EDTA)] complex was a polyhedron in shape (Figure 1B). The FT-IR spectra of this nano complex, as presented in Figure $1 \mathrm{C}$, confirmed the formation of the aforementioned complex as follows: a wide and strong band at $3000-3620 \mathrm{~cm}^{-1}$ centred at $3341 \mathrm{~cm}^{-1}$ could be attributed to $\mathrm{O}-\mathrm{H}$ vibrations. The peak at $1752 \mathrm{~cm}^{-1}$ was related to $\mathrm{C}=\mathrm{O}$ stretch band and at the $1637 \mathrm{~cm}^{-1}$ band could originate from the water molecules. The peak at $1637 \mathrm{~cm}^{-1}$ may also exhibit the presence of $\mathrm{Fe}-\mathrm{O}$ or could be related to EDTA carboxyl groups binding to surfaces. The peak at $1425 \mathrm{~cm}^{-1}$ could be attributed to the stretching vibration of $\mathrm{COO}^{-}$group (Nakamoto, 2009; Pavia et al., 2009).

\subsection{Plants treatment}

One group of plants received adequate $\mathrm{Fe}(20 \mu \mathrm{M}$ as $\mathrm{Fe}(\mathrm{II})$-EDTA) throughout the experiment until harvest (+Fe plants). The second group received low (5 $\mu \mathrm{M}) \mathrm{Fe}$ (low Fe plants) for 10 days, and in order to induce chlorosis, Fe was completely eliminated from the nutrient solution during the subsequent 20 days pre-culture period. After inducing severe chlorosis in the young leaves, this group was used for the application treatments.

In the root application (RA) treatment, low Fe plants were supplied with both nano or bulk Fe complexes (Fe(III)-EDTA) at $20 \mu \mathrm{M}$ through roots, whereas control plants were left untreated. In the foliar application (FA) treatment, nano and bulk Fe complexes were sprayed on the middle-aged, fully-expanded leaf (treated leaf), whereas the control plants were sprayed with distilled water. The Fe solution for foliar spray contained $0.4 \mathrm{mM}$ nano or bulk $\mathrm{Fe}$ complex at $\mathrm{pH}=$ 5.0 and was gradually sprayed within 5 days to avoid leaf damage. The final amount of Fe supplied to plants was similar for the RA and FA treatments $(22.4 \mathrm{mg}$ Fe plant ${ }^{-1}$ ).

Plants were cultured under controlled environmental conditions with a temperature regime of $25 / 18^{\circ} \mathrm{C}$ day/ night, 14/10 h light/dark period, a relative humidity of $50-60 \%$ and at a photon flux density of about 400 $\mu \mathrm{mol} \mathrm{m} \mathrm{m}^{-2} \mathrm{~s}^{-1}$.

\subsection{Harvest}

Plants were harvested one and two weeks after initiating the application treatments in parallel with corresponding $+\mathrm{Fe}$ plants. Between the two harvests, nutrient solution was not changed and the treated leaf of FA plants was sprayed with distilled water daily. At harvest, plants were thoroughly washed with doubledistilled water, blotted dry on filter paper and were divided into shoots and roots. In the FA plants, the shoots were divided into three fractions including treated leaf; middle-aged leaves, (ML), upper leaves (UL) corresponding to leaves above the treated including new leaves emerging after treatment commencement and lower leaves (LL), two leaves below the treated leaf). In analogy to the so-defined leaves in the FA treatment, the leaves of similar position in the RA treatment were separately harvested and subjected to further analyses.

\subsection{Determination of $\mathrm{Fe}$}

Leaf and root samples were dried at $70^{\circ} \mathrm{C}$ for 2 days, and after determining their dry weight, they were transferred to porcelain crucibles and dry-ashed at $550^{\circ} \mathrm{C}$ for $5 \mathrm{~h}$, resolved in $0.5 \mathrm{M} \mathrm{HCl}$ and were brought up to 
a final volume of $25 \mathrm{~mL}$ by adding double-distilled water. Fe concentration was determined by atomic absorption spectroscopy (Shimadzu, AA6300).

\subsection{Determination of Fe chlorophyll}

Leaf concentration of Chl was determined after extracting the pigments in cold acetone and allowing the samples to stand for $24 \mathrm{~h}$ in the dark at $4^{\circ} \mathrm{C}$ (Lichtenthaler and Wellburn, 1985). Leaf SPAD data were recorded daily after first $\mathrm{Fe}$ application until harvest.

\subsection{Calculation of parameters and statistical analyses}

Relative Fe content (\%) in each plant fraction was evaluated by the following equation:

$$
\text { Relative Fe content }=\frac{\text { Fe content in each fraction }(\mu \mathrm{g})}{\text { Total Fe content of analysed fractions }(\mu \mathrm{g})} \times 100 \quad \text { equation } 1
$$

Fe use efficiency (FeUE) was evaluated by the equation (2).
The biomass and $\mathrm{Fe}$ content of the analysed fractions were used to calculate this parameter.

$$
\text { FeUE }=\frac{\text { Plant biomass }(\mathrm{g})}{\text { Plant Fe content }(\mu \mathrm{g})}
$$

equation 2

The experiment was performed in complete randomised block design with four independent replica-

\section{Results}

Deficiency of Fe expectedly inhibited the plants' dry matter production. The plant biomass was not affected by Fe application one week after treatment initiation when the $+\mathrm{Fe}$, RA and FA plants were compared (Figure 2A). Comparing the biomass data within each application treatment, however, revealed a significant improvement in the root biomass by RA and in the shoot biomass by FA of both nano and bulk Fe complexes (Figure 2A). Two weeks after treatment, both RA and FA were efficient in improving the shoot and root growth, albeit nano Fe was more effective on shoot growth compared to bulk Fe when applied to the roots (Figure 2B). tions. Statistical analyses were carried out using Sigma Stat (3.02) with Tukey's post-hoc test $(\mathrm{P}<0.05)$.

SPAD data of the middle-aged leaf gradually increased after the application treatments; nano Fe was more effective than bulk Fe complex considering the rising SPAD readings in both RA (Figure $3 \mathrm{~A}$ ) and FA (Figure 3B). Comparing the two application modes revealed that, although RA increased the leaf SPAD quicker than LA, the same SPAD data were obtained two weeks after the treatment initiation, that is, 28.4 and 27.6 for nano Fe complex and 17.9-18.7 for bulk Fe complex in the RA and FA plants, respectively (Figure 3).

Leaf $\mathrm{Chl}$ concentration significantly responded to the root $\mathrm{Fe}$ application. In ML, RA of nano Fe complex was more efficient than the bulk Fe complex for increasing the Chl concentration; in LL, the bulk Fe complex was slightly more effective than the nano Fe complex. 
In UL, RA of both Fe complexes increased the Chl concentration to similar levels (Figure 4A).

Leaf $\mathrm{Fe}$ application increased the $\mathrm{Chl}$ concentrations of $\mathrm{UL}$ and ML (but not LL) in the -Fe plants one week after treatment (Figure 4A). Two weeks after first appli- cation of foliar $\mathrm{Fe}$, Chl concentrations were also increased in LL by both nano and bulk Fe spays. However, the enhancement of Chl was significantly higher when bulk Fe complex was supplied (Figure 4B).

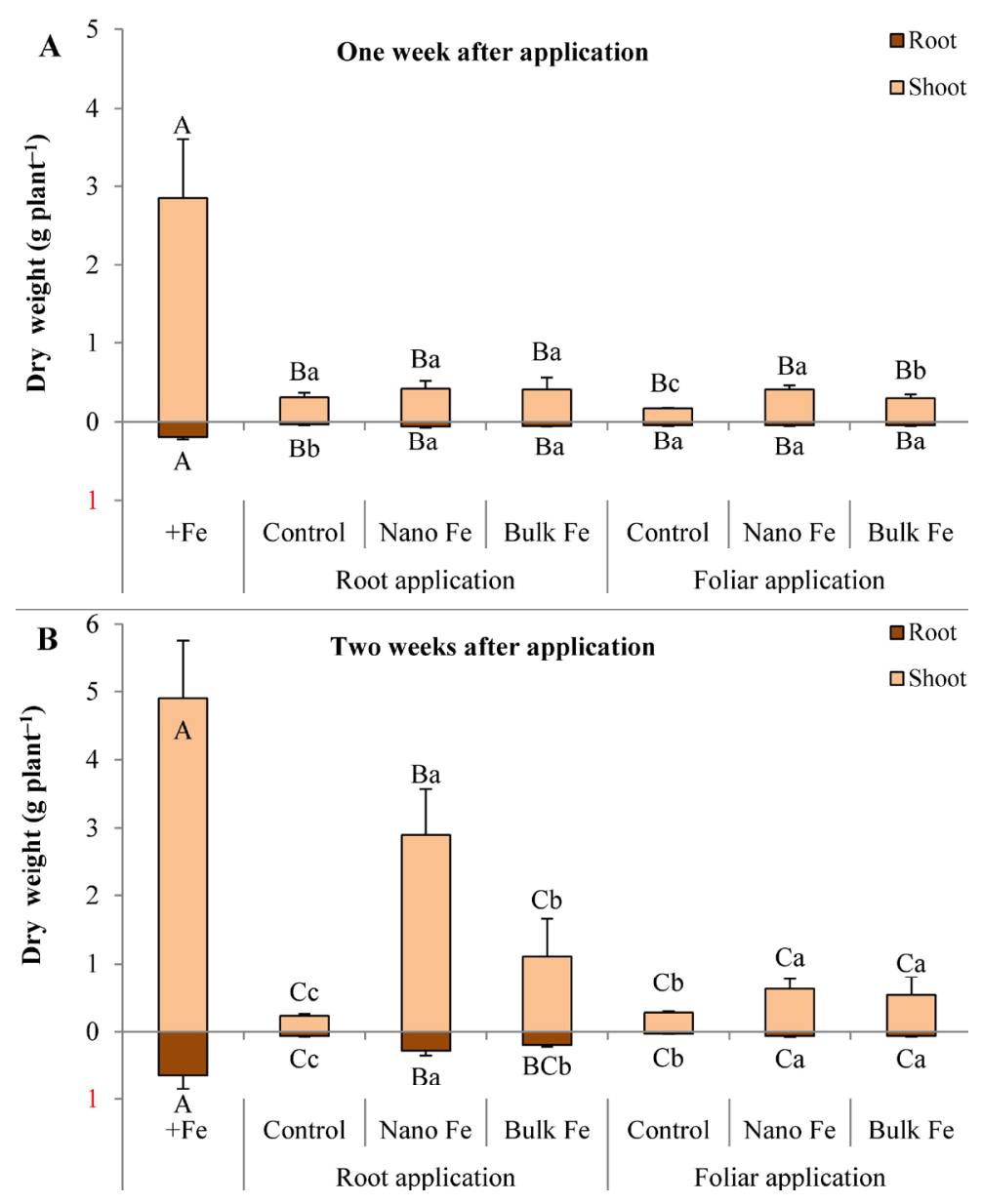

Figure 2. Shoot and root dry weight $\left(\mathrm{g}\right.$ plant ${ }^{-1}$ ) of tobacco (Nicotiana rustica L.) plants pre-cultured at low Fe supply for four weeks then either left untreated (Control) or treated with $\mathrm{Fe}$ as nano Fe or bulk Fe complex (Fe(III) EDTA) by application to roots or leaf. +Fe plants were treated with adequate Fe throughout the experiment. Bars indicated by the same upper case letters are not statistically different. Significant differences within each application treatment were indicated by different lower case letters $(\mathrm{P}<0.05)$. 

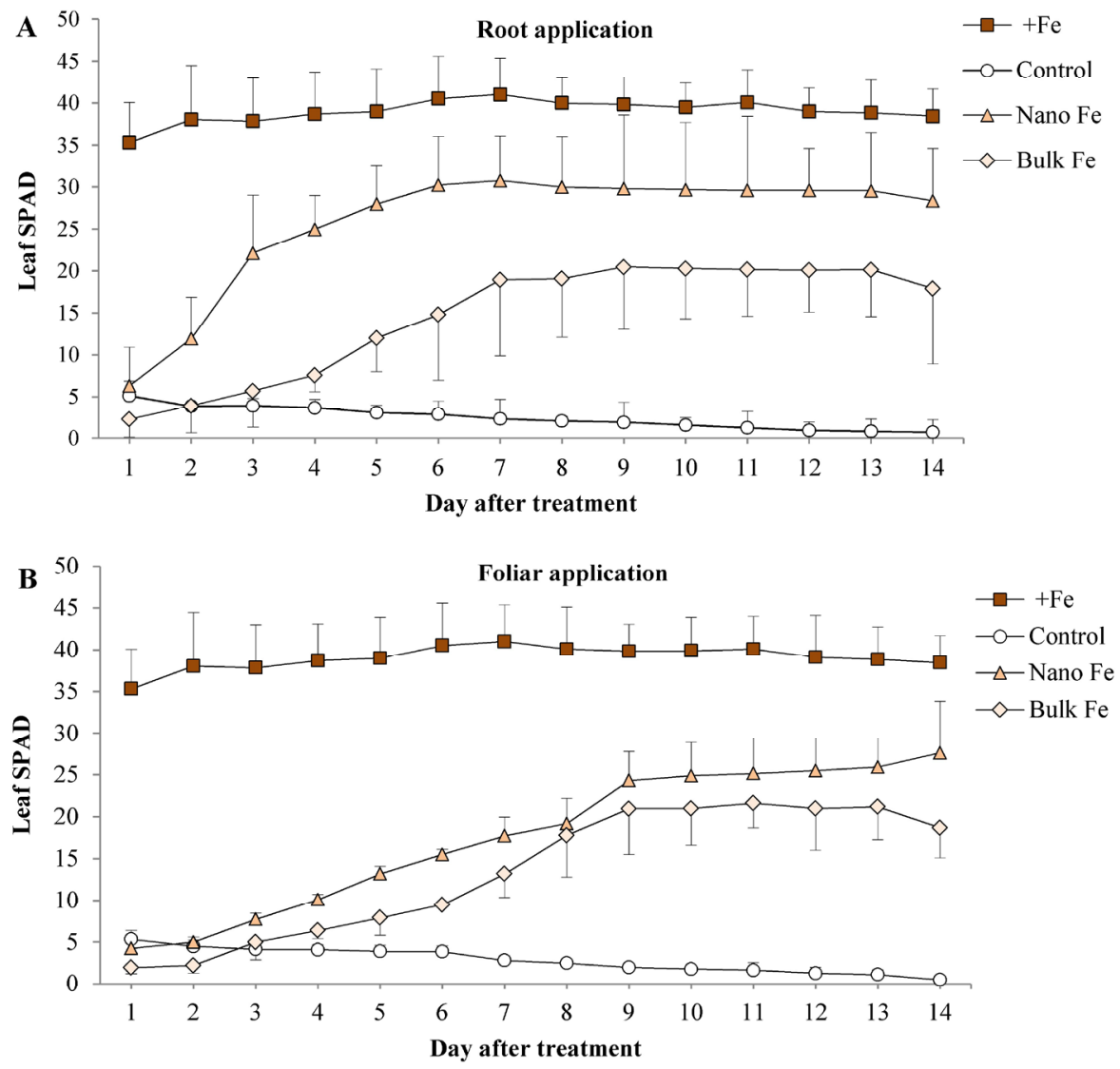

Figure 3. SPAD data of the middle-aged leaf in tobacco (Nicotiana rustica L.) plants pre-cultured at low Fe supply for four weeks then either left untreated (Control) or treated with Fe as nano Fe or bulk Fe complex (Fe(III) EDTA) by application to roots (A) or leaf (B). +Fe plants were treated with adequate Fe throughout the experiment. In the foliar application treatment, the middle-aged leaf was sprayed.

As expected, without Fe application the total $\mathrm{Fe}$ content was lower in the control plants than in the + Fe plants (Figure 5) and Fe supply enhanced the Fe content. One week after the start of the treatments the effect of bulk Fe was greater than that of nano Fe in both RA and FA plants (Figure 5A).
Two weeks after treatment, however, only FA plants revealed higher effect of the bulk Fe complex. Interestingly, Fe content of the RA plants was much greater than the FA ones two weeks after treatment without any difference between the two Fe complexes (Figure 5B). 

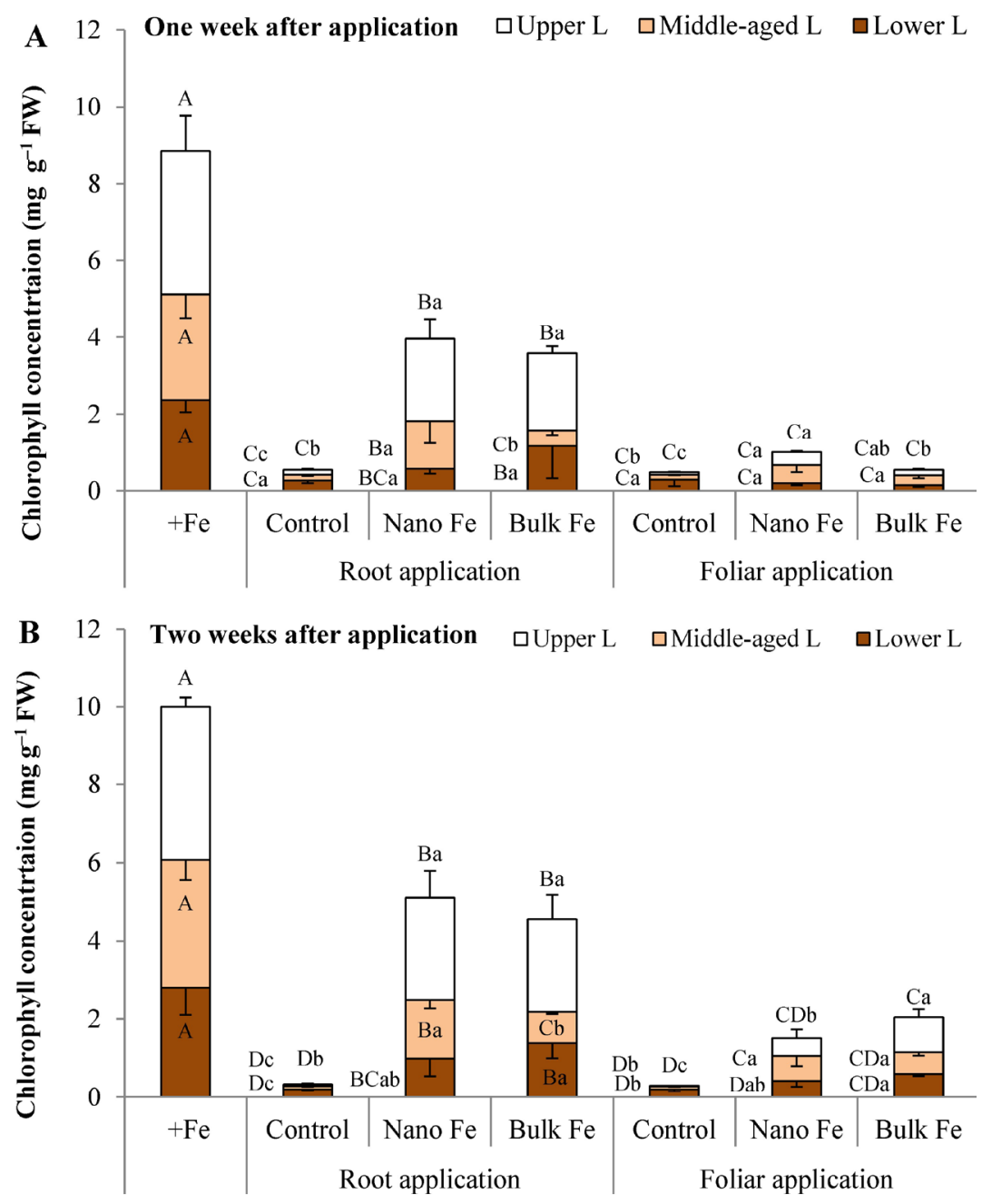

Figure 4. Leaf concentration of chlorophyll $\left(\mathrm{mg} \mathrm{g}^{-1} \mathrm{FW}\right)$ in the middle-aged leaf, the leaves located above (Upper L) and below it (Lower L) in tobacco (Nicotiana rustica L.) plants pre-cultured at low Fe supply for four weeks then either left untreated (Control) or treated with $\mathrm{Fe}$ as nano $\mathrm{Fe}$ or bulk Fe complex (Fe(III) EDTA) by application to roots or leaf. +Fe plants were treated with adequate Fe throughout the experiment. In the leaf application treatment, the middle-aged leaf was sprayed. Bars indicated by the same upper case letters are not statistically different. Significant differences within each application treatment were indicated by different lower case letters $(\mathrm{P}<0.05)$. 

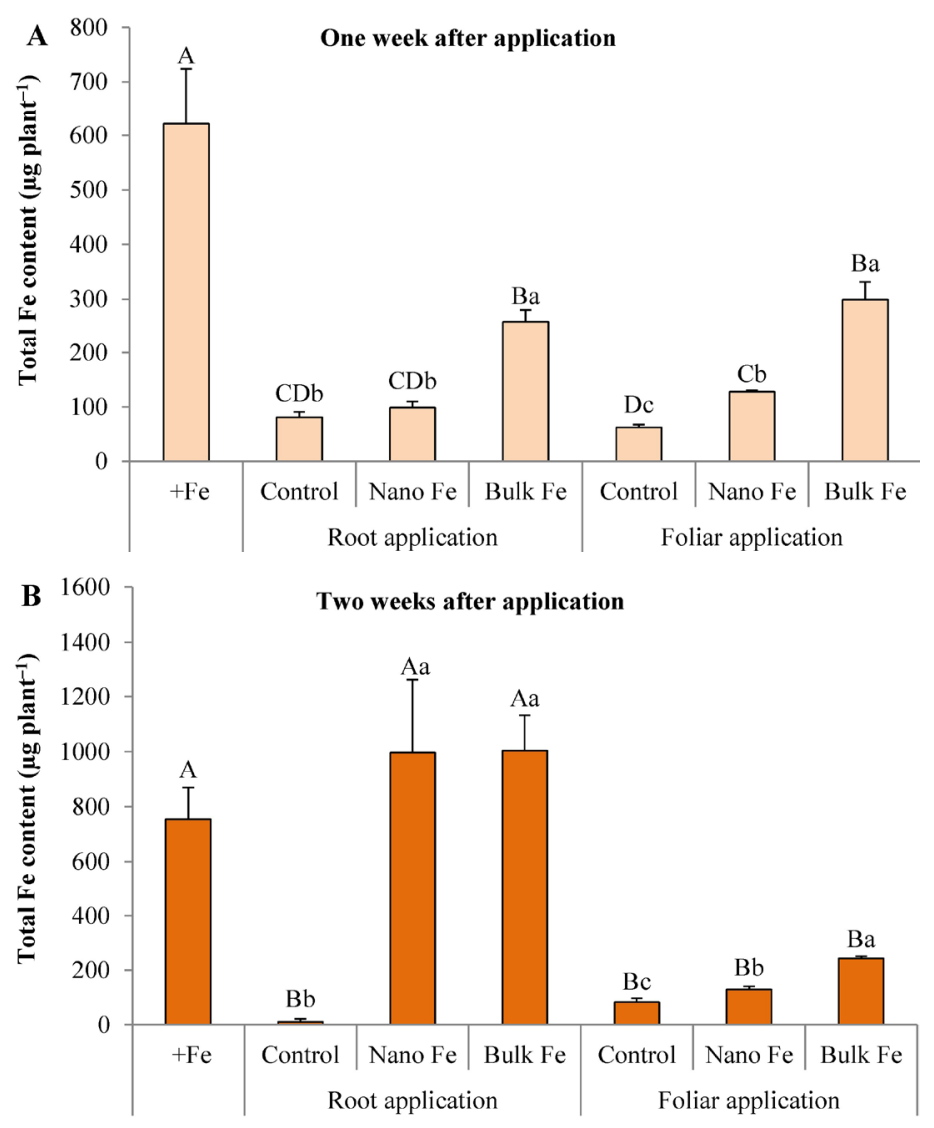

Figure 5. Total Fe content $\left(\mu\right.$ g plant $\left.^{-1}\right)$ in tobacco (Nicotiana rustica L.) plants pre-cultured at low Fe supply for four weeks then either left untreated (Control) or treated with Fe as nano Fe or bulk Fe complex (Fe(III)EDTA) by application to roots or leaf. $+\mathrm{Fe}$ plants were treated with adequate Fe throughout the experiment. Bars indicated by the same upper case letters are not statistically different. Significant differences within each application treatment were indicated by different lower case letters $(\mathrm{P}<0.05)$.

In the RA plants, Fe use efficiency (FeUE) of the control treatment was lower than $+\mathrm{Fe}$ plants, one week after treatment (Figure 6A). This effect disappeared in the plants harvested in the subsequent week due to biomass production without an increase in the Fe content (Figure 6B). Contrastingly, in the FA plants two weeks after treatment FeUE of the control plants was significantly higher than $+\mathrm{Fe}$ plants as a consequence of higher biomass production in the absence of Fe supply (Figure 6B). This effect likely resulted due to the water spray (see M\&M).

FeUE of the RA plants treated with nano Fe was significantly higher than those treated with bulk Fe complex at both harvest times (Figures 6A and 6B). In the FA plants, higher effect of nano Fe was observed than bulk Fe complex, only one week after treatment (Figure 6A). 

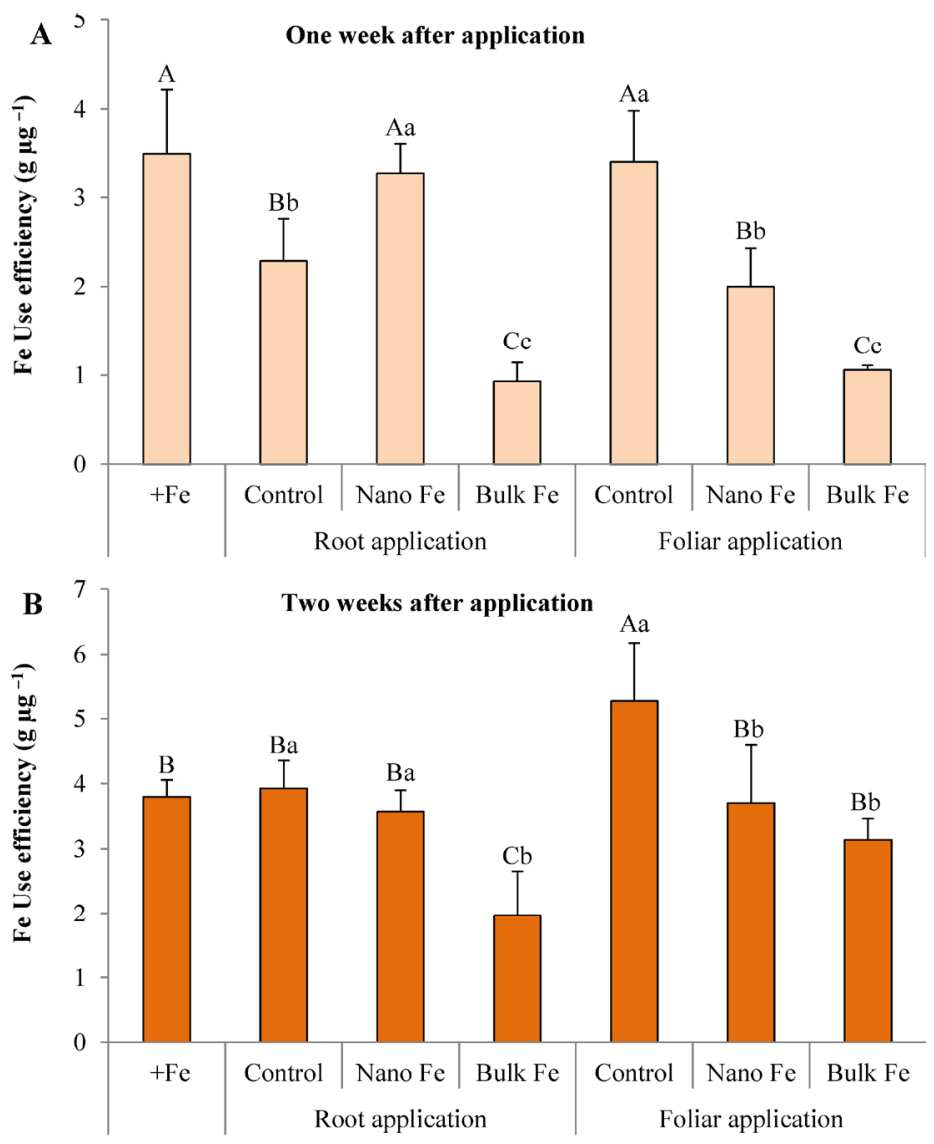

Figure 6. Iron use efficiency ( $\mathrm{g}^{-1}$ ) in tobacco (Nicotiana rustica L.) plants pre-cultured at low Fe supply for four weeks then either left untreated (Control) or treated with Fe as nano Fe or bulk Fe complex (Fe(III)EDTA) by application to roots or leaf. + Fe plants were treated with adequate Fe throughout the experiment. Bars indicated by the same upper case letters are not statistically different. Significant differences within each application treatment were indicated by different lower case letters $(\mathrm{P}<0.05)$.

In the Fe sufficient plants (+Fe plants), the majority of Fe was partitioned into UL and roots comprising about $80 \%$ of the plant total $\mathrm{Fe}$ content (Figure 7). Moreover, one week after treatment, the majority of applied $\mathrm{Fe}$ was found in the roots (of RA plants) or in the treated leaf (of FA plants). A considerable difference was also observed between the nano and bulk Fe complexes in the partitioning of applied $\mathrm{Fe}$, one week after treatment initiation.
Retention of Fe in the roots of the RA and in the treated leaf of the FA plants, were considerably higher when applied as bulk Fe compared with nano Fe complex (Figure 7A). Two weeks after treatment, however, this pattern changed, in particular, in the RA plants with the majority of Fe being partitioned into UL. In the FA plants, a slight reduction in the relative Fe content of the treated leaf was observed in the bulk Fe treatment (Figure 7B). 
Interestingly, Fe partitioning differed between the control treatment of the FA (sprayed with distilled water) and RA plants. In the control FA plants, wa- ter spray led to the Fe retention (originated from the pre-culture period) in ML (Figure 7).
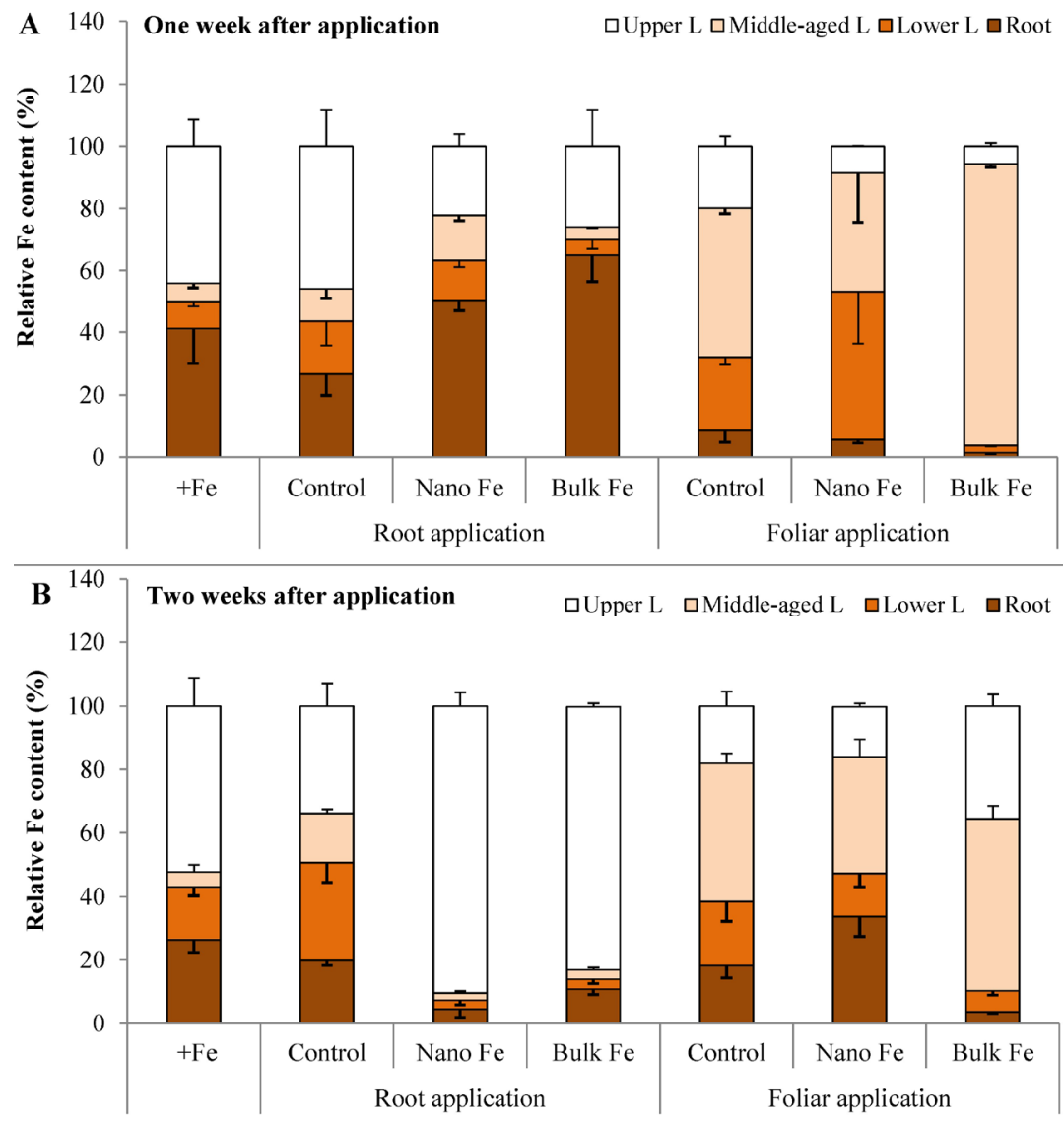

Figure 7. Relative Fe content (\%) in the middle-aged leaf, the leaves located above (Upper L) and below (Lower L) it and roots in tobacco (Nicotiana rustica L.) plants pre-cultured at low Fe supply for four weeks then either left untreated (Control) or treated with $\mathrm{Fe}$ as nano Fe or bulk Fe complex (Fe(III)EDTA) by application to roots or leaf. + Fe plants were treated with adequate Fe throughout the experiment. In the foliar application treatment, the middle-aged leaf was sprayed. 


\section{Discussion}

\subsection{Effect of Fe application on growth and leaf chlo- rophyll concentration}

Growth reduction is not the first symptom of Fe starvation, whereas chlorosis is the first and most obvious sign of Fe deficiency in many plant species. In the tobacco plants used in this study, however, the production of leaf chlorosis in the $-\mathrm{Fe}$ plants required for initiating the application treatment lasted for about four weeks, and consequently, a strong decline in plant growth occurred during the pre-culture treatment before Fe re-supply. Growth reduction is not only the consequence of reduction in the $\mathrm{Chl}$ synthesis, electron transport and $\mathrm{CO}_{2}$ assimilation in Fe-deficient plants. Other aspects of plant metabolism are also implied due to the contribution of $\mathrm{Fe}$ in the structure and activity of several heme and non-heme enzymes (Broadley et al., 2012; Kobayashi and Nishizawa, 2014).

Considering the leaf SPAD data in ML, we may conclude that the nano Fe complex was (slightly or significantly) more effective than the bulk Fe complex in retrieving the leaf $\mathrm{Chl}$ concentration in both RA and LA. This difference was more prominent in the RA plants at an earlier phase of Fe re-supply indicating higher efficiency of the nano Fe complex for correction of leaf chlorosis and prevention of decreased photosynthesis rate (see below). Leaf chlorosis is the most obvious visible symptom of Fe deficiency due to the direct contribution of $\mathrm{Fe}$ in the biosynthesis of $\mathrm{Chl}$ (Broadley et al., 2012; Briat et al., 2015; Mai et al., 2016). Comparison of $\mathrm{Chl}$ concentration of $+\mathrm{Fe}$ plants with the application treatments revealed that UL were more responsive to $\mathrm{Fe}$ application through roots than ML and LL without any difference between the two Fe compounds. In the FA treatment, in contrast, leaves of different age did not considerably differ in their response to Fe re-supply.

\subsection{Differences between nano and bulk Fe complexes} in the root-shoot translocation

Fe transport is a limiting step in Fe nutrition. After root uptake, $\mathrm{Fe}$ is radially transported across the root and then uploaded into the xylem. Xylem loading is a critical regulatory step for adequate Fe nutrition in plants. In the xylem, Fe is transported in chelated form, being citrate the main complexing agent involved (Lucena and Hernandez-Apaolaza, 2017). Comparison of $\mathrm{Chl}$ and $\mathrm{Fe}$ partitioning indicated differences between nano and bulk Fe complexes in the root-shoot translocation of the root-applied $\mathrm{Fe}$ in the tobacco plants of this work. One week after the treatment initiation, the majority of root-applied $\mathrm{Fe}$ was expectedly found in the roots. However, even after two weeks retention of $\mathrm{Fe}$ in the roots, was higher for the bulk Fe complex than nano Fe complex, indicating lower xylem mobility of the bulk Fe complex than nano Fe complex. Furthermore, one week after treatment, partitioning of root-derived $\mathrm{Fe}$ into $\mathrm{ML}$ and $\mathrm{LL}$ was higher for nano Fe complex when compared to the bulk Fe complex (Figure 7A); this was also reflected in the higher SPAD readings (Figure $3 \mathrm{~A}$ ) and $\mathrm{Chl}$ concentration of ML (Figure 4A). Although a majority of both Fe complexes were found in UL two weeks after treatment, the nano Fe complex was better partitioned into the growing young leaves than the bulk Fe complex. The better dry matter production in response to RA of nano Fe complex than of bulk Fe complex, could be, at least partly, attributed to the following: a faster root-shoot Fe translocation via xylem, more evenly Fe partitioning into the leaves of different age in a relatively short time span, and higher Fe partitioning into the young leaves during the subsequent growth period. Presumably, the higher surface area: volume ratio of nanoparticles (Monreal et al., 2015; Tripathi et al., 2017) makes them better than their bulk counterparts in terms of uptake and activity. 


\subsection{Different mobility of nano and bulk $\mathrm{Fe}$ in the phloem}

The distribution of nutrients, including Fe, to developing organs of plants depends to a great extent on phloem transport. Nicotianamine is one of the Fe chelator in plants which is involved in metal translocation in the plant body (Thomine and Vert, 2013). Remobilization and re-translocation of Fe via phloem to youngest leaves can reduce the severity of the chlorosis severity. However, an extremely poor redistribution via the phloem was found for Fe (Page and Feller, 2015). In the FA treatments, comparison of $\mathrm{Chl}$ concentration and $\mathrm{Fe}$ partitioning into the leaves of different age indicated differences between the phloem mobility of nano and bulk $\mathrm{Fe}$ in the tobacco plants used in this study. As observed one week after FA treatment, nano Fe was superior than bulk Fe complex in improving the Chl concentration of not only ML (treated leaf) but also UL and LL suggesting a higher phloem mobility of nano Fe than bulk Fe complex. This also reflected in the more evenly distributed nano $\mathrm{Fe}$ among leaves of different age (Figure 7A). The majority of leafapplied bulk Fe complex was retained in the treated leaf while nano Fe was considerably re-translocated, despite the short time span between the start of the application and the harvest. This emphasises the higher effectiveness of nano Fe complex for short-term correction of $\mathrm{Fe}$ deficiency-induced chlorosis. Two weeks after treatment, however, despite still higher retention in ML, a substantial re-translocation of bulk Fe complex into the sink leaves occurred. This was also reflected in the higher Chl concentration of UL and LL of the plants treated with the nano Fe than with bulk Fe complex. More evenly distributed nano Fe complex among different plant fractions observed in the FA plants likely resulted from its higher phloem mobility and/or higher xylem-phloem exchange in the FA leaves. Transfer cells with a proliferated cell surface are presumably key players for the selective xylem-to-phloem transfer of heavy metals and other solutes (Offler et al., 2003). In an experiment using $59 \mathrm{Fe}$ it has been observed that $\mathrm{Fe}$ is transported with the transpiration stream to minor leaf veins, followed by an accumulation in non-vascular tissues (Page and Feller, 2015) revealing a low xylem-phloem exchange for this element. In our work, however, probably due to its lower size, nano Fe was likely able to use this pathway more effectively than bulk Fe. Published data on the uptake and mobility of NPs by plants is still not conclusive and related information on the Fe nano complex is still missing. NPs can enter the plant cells by binding to carrier proteins, through aquaporins, ion channels or endocytosis The NPs may form complexes with the membrane transporters or root exudates and can subsequently be transported into the plants (Ghormade et al., 2011; Tripathi et al., 2017). Presumably, the nano Fe complex has higher ability to interact with transporters and the root ferric chelate reductase enzyme than bulk Fe; similar mechanism has been proposed as the basis for higher efficacy of Fe-chelates over Fe salts (Lucena and HernandezApaolaza, 2017). Higher mobility of nano Fe than bulk Fe complex in the xylem and phloem and less retention in the applied plant part could also be related to its lower precipitation in the root and leaf apoplast. Nano $\mathrm{Fe}$ is presumably less prone to be precipitated within plant apoplasm due to its lower size and higher mobility. Unfortunately, we did not determine the proportion of apoplastic and symplastic Fe in the tissues, thus, we could not evaluate this probability

\subsection{Different utilization of nano and bulk Fe applied to the roots}

In the RA treatments, nano $\mathrm{Fe}$ was more effectively used for biomass production than bulk Fe complex. Two weeks after treatment initiation, greater biomass 
production despite the same Fe content in plants supplied with nano compared with bulk Fe complex was observed. This indicates higher internal utilisation efficiency of the nano Fe complex (Baligar and Fageria, 2015). Our finding that plants receiving nano $\mathrm{Fe}$ complex through roots have higher FeUE opens the door to the development of new strategies allowing the reduction of the use of Fe fertilizers in agricultural production.

Unfortunately, the fate of nano Fe compounds and their mobility in soil, and its probable effect on soil biological properties in comparison with bulk $\mathrm{Fe}$ compounds still remains unclear. It has been stated that single NPs can enter into the soil micro-pores and if they are sorbed on mobile colloids, the mobility is highly enhanced. In contrast, aggregates of NPs remain in the soil macro-pores, and if they are sorbed on non-mobile particles, their mobility is inhibited (Ma et al., 2010). The behaviour of Fe NPs such as $\mathrm{Fe}_{3} \mathrm{O}_{4}$ is expected to be considerably different from that of Fe nano complexes.

\subsection{Different utilization of nano and bulk Fe applied to the leaves}

Similar to the RA plants, FeUE of the FA plants was higher in plants supplied with nano Fe complex than those treated with bulk Fe complex, one week after the start of the treatments. Higher FeUE values for plants treated with nano Fe complex resulted from both higher biomass and less Fe content. Presumably, less retention in the applied leaf and its more even distribution within the shoot allowed the whole plant for a quicker assimilation of nano Fe complex and its subsequent utilisation for biomass production. The advantage of nano over bulk Fe complex in biomass production, however, was not observed two weeks after treatment; this suggested that bulk Fe complex slowly but efficiently partitioned into the young growing leaves supporting growth of the whole plant.
Interestingly, $\mathrm{Fe}$ content significantly increased between the first and second harvest in the RA (but not in the LA) plants. This Fe obviously originated from the undepleted nutrient solution within the two weeks. The high Fe content in the RA plants harvested two weeks after treatment was most likely the consequence of the function of root mechanisms for $\mathrm{Fe}$ acquisition that are usually up-regulated under Fe starvation. The Fe transporter protein, IRT, is regulated both through transcriptional and post-translational events, governed by Fe nutritional status (Kobayashi and Nishizawa, 2012; 2014). It seems that root application of Fe is advantageous over foliar Fe spray due to the benefits of the Fe deficiency-induced responses in the roots. In the FA plants, in contrast, the lack of difference in the plant $\mathrm{Fe}$ content between the two harvest times implied that Fe deficiency did not result in the up-regulation of uptake mechanisms in the leaf cells. It is noteworthy that the Fe content supplied to the plants was similar for both RA and FA treatments (22.4 mg Fe plant ${ }^{-1}$ ), and in the FA plants, the treated leaves were not washed after foliar spray, instead, they were occasionally sprayed with distilled water in order to dissolve the dried Fe solution on the leaf surfaces.

\section{Conclusions}

In this study, root application (RA) of Fe was considerably more effective than foliar application (FA) for the biomass production. This seems due to the fact that root-applied Fe was chiefly translocated to the new growing leaves that are essential for supporting the whole plant growth. Higher effectiveness of nano Fe for biomass production than bulk Fe complex was due to the higher nano Fe partitioning into the young growing leaves. Another reason for greater effectiveness of root-applied $\mathrm{Fe}$ was the benefit from the Fedeficiency induced enhancement of Fe uptake capacity in the roots under Fe starvation. 
Foliar application however, could also restore $\mathrm{Chl}$ concentrations to the same extent as RA. This was confirmed by the similar final SPAD data of the RA and FA plants for the leaves with direct spray on middle-aged leaves (ML) at the end of experiment. Our results suggested that FA is more effective than RA in extending the green leaf area duration.

Nano Fe complex is an advantageous form of Fe supply both as a leaf spray for an immediate response and as a long-term $\mathrm{Fe}$ feeder of plant through roots. Before its recommendation as an Fe fertilizer however, more information is needed on its precipitation, mobility and environmental consequences of its presence in the soil.

\section{Acknowledgments}

The authors would like to thank Dr. Charlotte Poschenrieder, Autonomous University of Barcelona, Spain for the critical reading and correction of the manuscript. Research Council and the Center of Excellence for Biodiversity, University of Tabriz are greatly acknowledged for financial supports.

\section{References}

Baligar, V.C., Fageria, N.K. 2015. Nutrient use efficiency in plants: An overview. In: A. Rakshit, H. B. Singh, A. Sen (eds). Nutrient Use Efficiency: From Basics to Advances. Springer, India, pp. 1-14.

Briat, J.F., Dubos, C., Gaymard, F. 2015. Iron nutrition, biomass production, and plant product quality. Trends Plant Sci. 20, 33-40.

Broadley, M., Brown, P., Kakmak, I., Rengel, Z., Zhao, F. 2012. Function of nutrients: Micronutrients. In: P. Marschner (ed). Marschner's MineralNutrition of Higher Plants, Academic Press, London, pp. 71-84.
Granja, F., Covarrubias, J.I. 2018. Evaluation of acidifying nitrogen fertilizers in avocado trees with iron deficiency symptoms. J Soil Sci. Plant Nutr. $18,157-172$.

Golshahi, S., Ahangar, A.G., Mir, N., Ghorbani, M. 2018. A comparison of the use of different sources of nanoscale iron particles on the concentration of micronutrients and plasma membrane stability in sorghum. J Soil Sci. Plant Nutr. 18, 236-252.

Eichert, T., Fernandez, V. 2012. Uptake and release of elements by leaves and other aerial plant. Evidence from measurement of diffusion potentials. Plant Physiol. 92, 103-109.

Fernández, V., Brown, P.H. 2013. From plant surface to plant metabolism: the uncertain fate of foliarapplied nutrients. Front. Plant Sci. 4, 289.

Fernandez, V., Del Rio, V., Pumarino, L., Igartua, E., Abadia, J., Abadia, A. 2008. Foliar fertilization of peach (Prunus persica (L.) batsch) with different iron formulations: Effects on re-greening, iron concentration and mineral composition in treated and untreated leaf surfaces. Sci. Hort. 117, 241-248.

Fernandez, V., Ebert, G. 2005. Foliar iron fertilization - A critical review. J. Plant Nutr. 28, 2113-2124.

Ghormade, V., Deshpande, M.V., Paknikar, K.M. 2011. Perspectives for nano-biotechnology enabled protection and nutrition of plants. Biotechnol. Adv. 29, 792-803.

Jalali, M., Ghanati, F., Modarres-Sanavi, A. M. 2016. Effect of $\mathrm{Fe} 3 \mathrm{O} 4$ nanoparticles and iron chelate on the antioxidant capacity and nutritional value of soil-cultivated maize (Zea mays) plants. Crop Pasture Sci. 67, 621-628.

Jalali, M., Ghanati, F., Modarres-Sanavi, A.M., Khoshgoftarmanesh, A.H. 2017. Physiological effects of repeated foliar application of magnetite nanoparticles on maize plants. J. Agron. Crop Sci. 203, 593-602. 
Jeyasubramanian, K., Thoppey, U.U., Hikku, G.S., Selvakumar, N., Subramania, A., Krishnamoorthy, K. 2016. Enhancement in growth rate and productivity of spinach grown in hydroponics with iron oxide nanoparticles. RSC Adv. 6, 15451-15459.

Johnson, C.M., Stout, P.R., Broyer, T.C. 1957. Carlton AB. Comparative chlorine requirements of different plant species. Plant Soil. 8, 337-353.

Kannan, S. 2010. Foliar fertilization for sustainable crop production. Sustain. Agric. Rev. 4, 371-402.

Kobayashi, T., Nishizawa, N.K. 2012. Iron uptake, translocation, and regulation in higher plants. Annu. Rev. Plant Biol. 63, 131-152.

Kobayashi, T., Nishizawa, N.K. 2014. Iron sensors and signals in response to iron deficiency. Plant Sci. 224, 36-43.

Lichtenthaler, H.K., Wellburn, A.R. 1983. Determination of total carotenoids and chlorophylls $\mathrm{a}$ and $\mathrm{b}$ of leaf in different solvents. Biol. Soc. Trans. 11, 591-592.

Lucena, J.J., Hernandez-Apaolaza, L. 2017. Iron nutrition in plants: an overview. Plant Soil. 418, 1-4.

Ma, X., Geiser-Lee, J., Deng, Y., Kolmakov, A. 2010. Interactions between engineered nanoparticles (ENPs) and plants: phytotoxicity, uptake and accumulation. Sci. Total Environ. 408, 3053-3061.

Mai, H.J., Bauer, P. 2016. From the proteomic point of view: Integration of adaptive changes to iron deficiency in plants. Current Plant Biol. 5, 45-56.

Márquez-Quiroz, C., De-la-Cruz-Lázaro, E., OsorioOsorio, R., Sánchez-Chávez, E. 2015. Biofortification of cowpea beans with iron: iron's influence on mineral content and yield. J. Soil Sci. Plant Nutr. 15, 839-847.

Monreal, C.M. DeRosa, M., Mallubhotla, S.C., Bindraban, P.S., Dimkpa, C. 2015. The Application of Nanotechnology for Micronutrients in Soil-Plant
Systems. VFRC (Virtual Fertilizer Research Center) Report, Washington, DC, USA, 53 p.

Nakamoto, K. 2009. Infrared and Raman Spectra of Inorganic and Coordination Compounds: Part B: Applications in Coordination, Organometallic, and Bioinorganic Chemistry, 6th Edition. John Wiley \& Sons, Inc, USA.

Offler, C.E., McCurdy, D.W., Patrick, J.W., Talbot, M.J. 2003. Transfer cells: cells specialized for a special purpose. Annu. Rev. Plant Biol. 54, 431454.

Page, V., Feller, U. 2015. Heavy metals in crop plants: Transport and redistribution processes on the whole plant level. Agronomy. 5, 447-463.

Pavia, D.L., Gary M. Lampman, G.M., Kriz, G.S., Vyvyan, J.A. 2009. Introduction to Spectroscopy, 5 th edition. Cengage Learning, USA.

Rengel, Z. 2015. Availability of Mn, Zn and Fe in the rhizosphere. J. Soil Sci. Plant Nutr. 15, 397-409.

Rios, J.J., Carrasco-Gil, S., Abadía, A., Abadía, J. 2016. Using Perls staining to trace the iron uptake pathway in leaves of a Prunus rootstock treated with iron foliar fertilizers. Front. Plant Sci. 7, 893.

Thomine, S., Vert, G. 2013. Iron transport in plants: better be safe than sorry. Curr. Opin. Plant Biol. $16,322-327$.

Tripathi, D.K., Singh, S., Singh, S., Pandey, R., Singh, V.P., Sharma, N. C., Prasad, S.M., Dubey, N.K., Chauhan, D.K. 2017. An overview on manufactured nanoparticles in plants: uptake, translocation, accumulation and phytotoxicity. Plant Physiol. Biochem. 110, 2-12.

Yadegari, M. 2015. Foliar application of micronutrients on essential oils of borago, thyme and marigold. J. Soil Sci. Plant Nutr. 15, 949-964. 
Zhu, H., Han, J., Xiao, J.Q., Jin, Y. 2008. Uptake, translocation, and accumulation of manufactured iron oxide nanoparticles by pumpkin plants. J. Environ. Monit. 10, 713-717.
Zuo, Y., Zhang, F. 2011. Soil and crop management strategies to prevent iron deficiency in crops Plant Soil. 339, 83-95. 